\title{
Electron densities in the upper ionosphere of Mars from the excitation of electron plasma oscillations
}

\author{
F. Duru, ${ }^{1}$ D. A. Gurnett, ${ }^{1}$ D. D. Morgan, ${ }^{1}$ R. Modolo, ${ }^{1}$ A. F. Nagy, ${ }^{2}$ and D. Najib ${ }^{2}$
}

Received 1 February 2008; revised 20 February 2008; accepted 28 February 2008; published 9 July 2008.

[1] In addition to remote radio sounding of the ionosphere of Mars, the MARSIS (Mars Advanced Radar for Subsurface and Ionospheric Sounding) instrument on the Mars Express spacecraft is also able to measure the in situ electron density from the excitation of local electron plasma oscillations. This paper presents an investigation of the electron density in the upper ionosphere of Mars based on the frequency of these oscillations. The advantage of this method is that electron densities can be measured at much higher altitudes than can be determined from remote radio soundings. Using this technique electron densities from 503 orbits have been analyzed over the period from 4 August 2005 to 31 July 2007 for altitudes ranging from about 275 to $1300 \mathrm{~km}$. Although there is considerable variability from orbit to orbit, the median electron density at a given solar zenith angle (SZA) on the dayside of Mars decreases systematically with increasing altitude with a characteristic plasma scale height varying from about 80 to $145 \mathrm{~km}$. At a fixed altitude, the electron density remains almost constant for SZAs less than about $80^{\circ}$. For SZAs greater than about $80^{\circ}$ the electron density decreases rapidly with increasing SZA, approaching very low values on the nightside. Simulations performed using both magnetohydrodynamic and hybrid codes show that the nearly constant density at a given altitude is caused by the horizontal transport of plasma from the dayside toward the nightside due to interaction with the solar wind.

Citation: Duru, F., D. A. Gurnett, D. D. Morgan, R. Modolo, A. F. Nagy, and D. Najib (2008), Electron densities in the upper ionosphere of Mars from the excitation of electron plasma oscillations, J. Geophys. Res., 113, A07302, doi:10.1029/2008JA013073.

\section{Introduction}

[2] The Mars Express spacecraft, which was placed in a highly eccentric orbit around Mars on 25 December 2003 [Chicarro et al., 2004], carries a low-frequency radar called MARSIS (Mars Advanced Radar for Subsurface and Ionospheric Sounding) that is designed to perform radar soundings of the subsurface and the ionosphere of Mars [Picardi et al., 2004]. Prior to Mars Express, most of the information about the ionosphere of Mars was obtained from radio occultation measurements [Kliore et al.,1965; Fjeldbo et al., 1966; Zhang et al., 1990; Luhmann and Brace, 1991; Kliore, 1992; Hinson et al., 2001; Patzold et al., 2005]. The radio occultation technique is based on measurements of the phase shift of a radio signal from a spacecraft as it is occulted by the ionosphere of a planet [Schunk and Nagy, 2000]. The resulting variations in the phase shift can be analyzed to give the vertical distribution of the electron density in the ionosphere. Mariner 4 provided the first radio occultation measurements of the ionosphere at Mars [Kliore

\footnotetext{
${ }^{1}$ Department of Physics and Astronomy, University of Iowa, Iowa City, Iowa, USA.

${ }^{2}$ Department of Atmospheric, Oceanic and Space Sciences, University of Michigan, Ann Arbor, Michigan, USA.

Copyright 2008 by the American Geophysical Union. 0148-0227/08/2008JA013073
}

et al., 1965]. The first direct measurements of the ions in the ionosphere of Mars were obtained by the Retarding Potential Analyzers on Viking 1 and 2 [Hanson et al., 1977]. More recently, MARSIS provided the first measurements of the electron density in the Martian ionosphere using the radar sounding technique [Gurnett et al., 2005].

[3] Radar soundings are performed by transmitting a short pulse of radio waves at a fixed frequency, $\mathrm{f}$, and then measuring the time delay, $\Delta t$, of the echo reflected from the top side of the ionosphere [Gurnett et al., 2005]. For vertical incidence on a horizontally stratified ionosphere, reflection occurs at the height in the ionosphere where the wave frequency is equal to the electron plasma frequency, which is given by

$$
\mathrm{f}_{\mathrm{p}}=8980 \sqrt{\mathrm{n}_{\mathrm{e}}}
$$

where $\mathrm{f}_{\mathrm{p}}$ is the plasma frequency in $\mathrm{Hz}$ and $\mathrm{n}_{\mathrm{e}}$ is the electron density in $\mathrm{cm}^{-3}$. In order to obtain a vertical profile of the electron density, the transmission frequency is stepped through a series of contiguous frequencies in order to give the time delay, hence range, to the reflection point as a function of frequency. Well known mathematical techniques can be used to correct the range for the dispersion caused by the plasma [Budden, 1961]. Using these techniques the time delays as a function of frequency can be converted into electron density as a function of height. One of the 
advantages of the radar sounding method compared to the radio occultation method is that it provides coverage of solar zenith angles (SZA) that cannot be obtained from radio occultations. It also provides much higher horizontal spatial resolution, thereby allowing the study of small scale structures in the ionosphere [Gurnett et al., 2005, 2008; Duru et al., 2006].

[4] In addition to remote radio sounding, electron densities can also be obtained from MARSIS by measuring the frequency of local electron plasma oscillations excited by the radar transmitter. As the transmitter steps in frequency, strong local electrostatic plasma oscillations, also called Langmuir waves [Gurnett and Bhattacharjee, 2005], are excited as the transmitter frequency passes through the local electron plasma frequency, i.e., when $f=f_{p}$. From the frequency of the oscillation the local electron density can then be calculated using equation (1). The purpose of this paper is to present a study of the electron density in the Martian ionosphere based on the excitation of electron plasma oscillations. As we will show, this technique can provide measurements at much higher altitudes than is possible from radio occultation measurements or the MARSIS remote radar soundings.

[5] The MARSIS instrument consists of a $40 \mathrm{~m}$ tip-to-tip electric dipole antenna, a $7 \mathrm{~m}$ monopole antenna, a radio transmitter, a receiver, and a digital signal processing system. For the normal ionospheric sounding mode used by MARSIS the transmitter steps through 160 quasilogarithmically spaced frequencies $(\Delta \mathrm{f} / \mathrm{f} \approx 2 \%)$ from $100 \mathrm{kHz}$ to $5.5 \mathrm{MHz}$. At each step a $91 \mu \mathrm{s}$ quasi-sinusoidal pulse is transmitted at a fixed frequency and the intensity of the resulting echoes are recorded by a digital receiver in 80 equally spaced time delay bins over an interval of $7.31 \mathrm{~ms}$. The receiver has a bandwidth of $10.9 \mathrm{kHz}$ centered on the frequency of the transmitted pulse. A complete scan through all 160 frequencies takes $1.26 \mathrm{~s}$, and the basic sweep cycle is repeated once every $7.54 \mathrm{~s}$. For a complete description of the MARSIS instrument see Picardi et al. [2004].

[6] Initially, the Mars Express orbit had a periapsis altitude of $275 \mathrm{~km}$, an apoapsis altitude of about $11,000 \mathrm{~km}$ and a period of about $6.75 \mathrm{~h}$ [Chicarro et al., 2004]. Because of gravitational and other perturbations these orbital parameters have changed somewhat during the course of the mission. Because the orbit is highly eccentric and the radar range is limited to not much more than a thousand kilometers, radar measurements can only be obtained in the region around periapsis. A typical ionospheric sounding pass starts at an inbound altitude of about $1300 \mathrm{~km}$, extends through periapsis at an altitude of about $275 \mathrm{~km}$, and ends at an outbound altitude of about $1300 \mathrm{~km}$. The data analyzed in this paper cover the period from 4 August 2005, to 31 July 2007. During this period data from 503 full or nearly full periapsis passes were investigated. Although most of the ionospheric sounding passes are centered on periapsis and last around $40 \mathrm{~min}$, some are restricted by subsurface sounding requirements to short 5 to 10 min inbound or outbound segments at very high altitudes, mostly on the nightside. Figure 1 shows the altitude and solar zenith angle coverage of all the data analyzed. It should be noted that there are some regions that are sparsely covered, and other regions with overlapping data from markedly different time intervals, in some cases more than a year apart. Because of the limited duration of the data, it was not possible to cover a full solar cycle in this study. The data were obtained in a time period with moderate to low solar activity. As more data are obtained in the extended mission, it may be possible to study solar cycle effects.

\section{Identification of the Local Electron Plasma Frequency}

[7] As discussed earlier, in the ionospheric sounding mode, the sounder transmitter excites electrostatic oscillations in the surrounding plasma as the sounding frequency is stepped through the local electron plasma frequency. Since the electron plasma oscillations are usually very intense, with antenna voltages much greater than the preamplifier power supply voltage, the received waveforms are often severely clipped. The resulting distortion then introduces harmonics at multiples of the basic oscillation frequency. To analyze the received signals the data are usually displayed in the form of an ionogram, which is a threedimensional color-coded plot of the received intensity as a function of frequency, $f$, on the horizontal axis, and delay time, $\Delta t$, on the vertical axis. An example of a MARSIS ionogram is shown in Figure 2. The first vertical line at the very left of the ionogram is caused by the excitation of electron plasma oscillations and is at the local electron plasma frequency, $f_{p}$. The other equally spaced vertical lines at higher frequencies are the harmonics generated by distortion in the preamplifier. If the plasma frequency is below the lower limit of the frequency of the receiver, i.e., less than $100 \mathrm{kHz}$, which often happens at high altitudes, the fundamental of the electron plasma frequency cannot be observed. However, the electron densities can still be determined by measuring the spacing of the harmonics [Gurnett et al., 2005, 2008]. This unanticipated consequence of the preamplifier distortion allows measurements of electron densities as low as $20 \mathrm{~cm}^{-3}$ at very high altitudes, well below the densities that can be obtained from the remote radar soundings. To determine the electron plasma frequency from an ionogram, an electronic ruler with an adjustable frequency spacing is used. To measure the plasma frequency the evenly spaced tick marks on the ruler are brought to the centers of the corresponding harmonic lines in the ionogram. The short vertical black lines at the top the ionogram in Figure 2 are the tick marks after they have been adjusted to coincide with the harmonic lines. Our experience is that the electron plasma frequency can be measured with an accuracy of about $\pm 1 \%$, which corresponds to an accuracy for measuring the electron density of about $\pm 2 \%$.

[8] When calculating the plasma frequency we assume that the oscillation frequency is at exactly the electron plasma frequency, thereby ignoring thermal effects, as in cold plasma theory. The possible importance of thermal effects can be analyzed by using the Bohm-Gross dispersion relation, which gives the oscillation frequency including thermal corrections [Gurnett and Bhattacharjee, 2005]. The Bohm-Gross dispersion relation is given by

$$
\omega^{2}=\omega_{\mathrm{pe}}^{2}\left[1+3 \lambda_{\mathrm{De}}^{2} \mathrm{k}^{2}\right]
$$




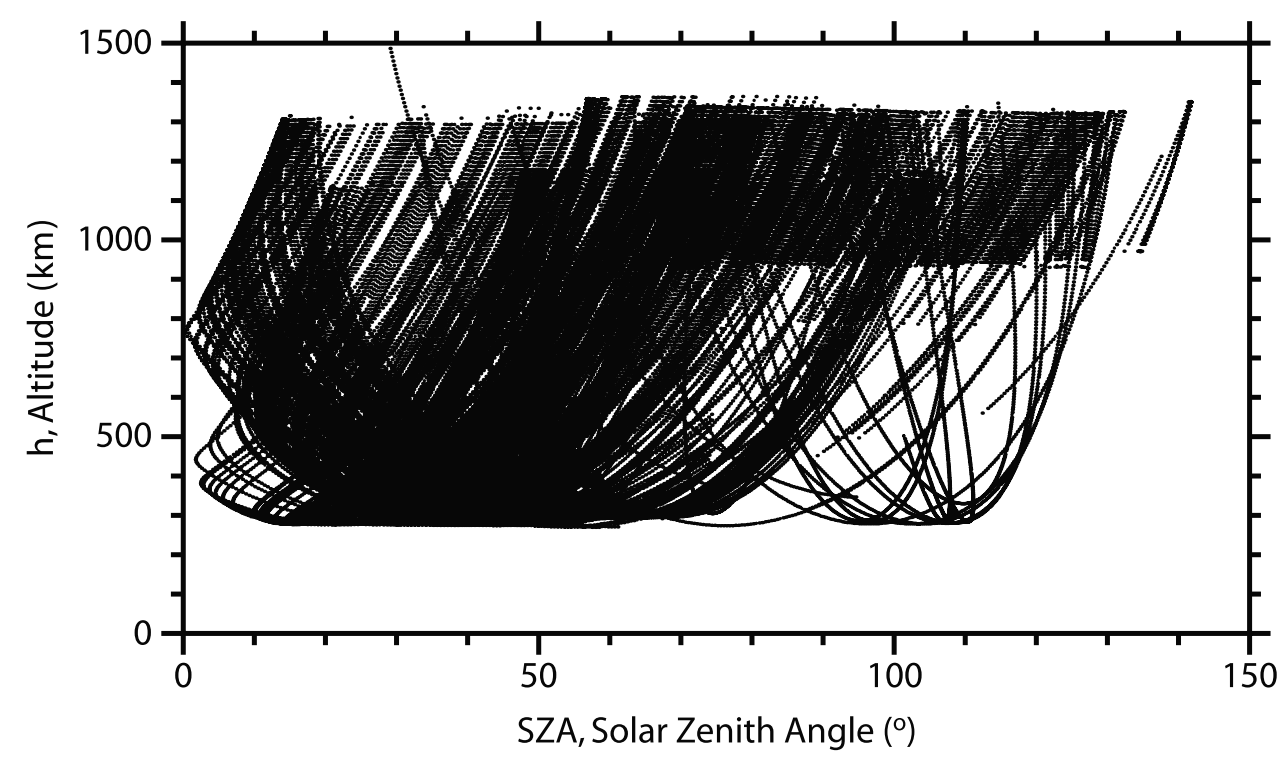

Figure 1. Coverage of MARSIS data as a function of altitude and solar zenith angle. Sampling density is quite uneven. In some regions different passes overlap, whereas, in some other regions there are no data at all. The inhomogeneities are believed to add to the irregularities in the results.

where $\lambda_{\mathrm{De}}$ is the Debye length, $\mathrm{k}=2 \pi / \lambda$ is the wave number, and $\lambda$ is the wavelength. The thermal correction is given by the second term on the right-hand side of the above equation. To see that this term is generally small we must compare the wavelength of the plasma oscillations to the Debye length. For a dipole antenna, like the one used on MARSIS, the dominant wavelength excited, $\lambda$, is approximately the tip-to-tip length of the antenna, which is $\mathrm{L}=40 \mathrm{~m}$.

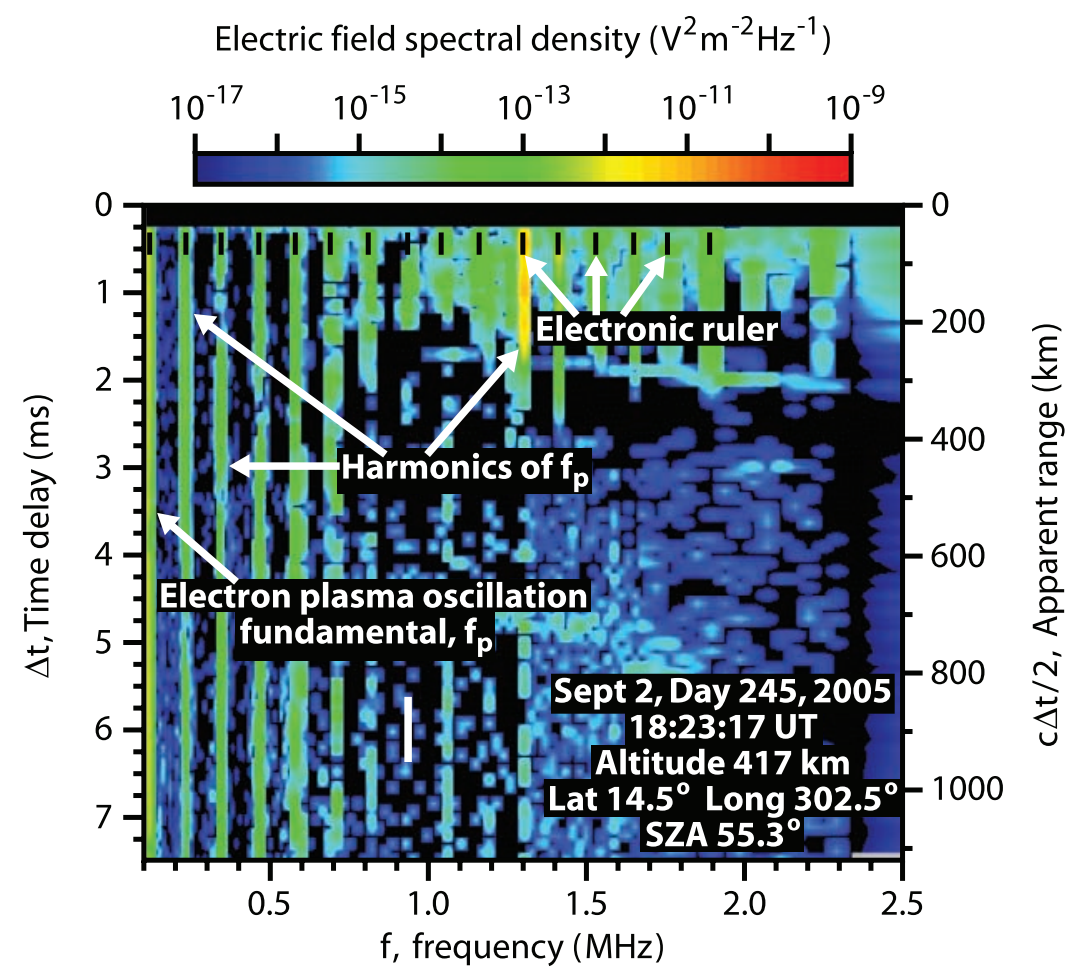

Figure 2. An example of an ionogram, which is a three-dimensional plot of received intensity as a function of time delay and frequency. The first vertical line around $0.1 \mathrm{MHz}$ is the local electron plasma frequency, $f_{p}$, and the other equally spaced vertical lines are the harmonics of the local electron plasma frequency. The short black lines superimposed on the green vertical lines are the tick marks of the electronic ruler. The spacing between these tick marks can be adjusted to match the spacing between the harmonics, which in turn gives the local electron plasma frequency. 
The Debye length is given by $\lambda_{\text {De }}=6.9\left(\mathrm{~T}_{\mathrm{e}} / \mathrm{n}_{\mathrm{e}}\right)^{1 / 2} \mathrm{~cm}$, where $\mathrm{T}_{\mathrm{e}}$ is the temperature in degrees Kelvin and $\mathrm{n}_{\mathrm{e}}$ is the electron number density in $\mathrm{cm}^{-3}$ [Gurnett and Bhattacharjee, 2005]. To estimate the largest Debye length that is likely to be encountered in the Martian ionosphere, $T_{e}$ is taken to be $5000 \mathrm{~K}$, which is an upper limit on electron temperature in the Martian ionosphere [Nagy et al., 2004], and $\mathrm{n}_{\mathrm{e}}$ is taken to be $10 \mathrm{~cm}^{-3}$, a lower limit on the electron density that can be measured by our technique. For these parameters, the maximum Debye length is $\lambda_{\mathrm{De}}=1.5 \mathrm{~m}$. The thermal correction in equation (2) is then $3\left(\lambda^{2}{ }_{\text {De }} \mathrm{k}^{2}\right)=0.176$, which corresponds to a frequency shift of about $8.4 \%$. This frequency shift is less than the frequency resolution of the receiver at low frequencies, which is $\Delta \mathrm{f} / \mathrm{f} \sim 10 \%$ for a density of $10 \mathrm{~cm}^{-3}$. At higher densities and lower temperatures, where the Debye length is even smaller, the thermal correction is completely negligible.

[9] From inspection of the MARSIS ionograms, we have found that in some regions, especially at high altitudes, plasma oscillations (or their harmonics) often cannot be detected. The transition into and out of such regions is often very abrupt. There are three reasons why plasma oscillations might not be detected. The first is that the electron density is so low that the fundamental oscillation frequency and the resulting harmonics are below the $100 \mathrm{kHz}$ low frequency limit of the receiver. Since harmonic numbers greater than about 10 are typically very weak, this limitation places a lower limit of about $10 \mathrm{kHz}$ on the electron plasma frequency that can produce detectable harmonics. From equation (1) this limit is seen to correspond to an electron density of only about $1 \mathrm{~cm}^{-3}$. However, for such low densities the harmonic spacing is less than the frequency resolution of the instrument, $10.9 \mathrm{kHz}$, so that even if the harmonics are present, they cannot be resolved. Our experience in analyzing the MARSIS ionograms is that the harmonic spacing must be at least $30 \mathrm{kHz}$ before the harmonics can be reliably identified. This spacing corresponds to a minimum detectable electron density of about $10 \mathrm{~cm}^{-3}$.

[10] The second reason that plasma oscillations (or their harmonics) might not be detected is that the spacecraft is in a region with a very large plasma flow velocity. If the plasma flow velocity is very large, the highly localized wave packet excited by the sounder is swept away from the spacecraft before the receiver can respond. To produce a response in the receiver the wave packet must remain in the vicinity of the spacecraft for a minimum of about $\Delta \mathrm{t}(\mathrm{min})=0.25 \mathrm{~ms}$, which is the minimum time delay that can be resolved (see the time delay scale on the ionogram in Figure 2). Since the scale size of the wave packet excited by the sounder is approximately $\mathrm{L}=40 \mathrm{~m}$, as a rough estimate we can say that plasma oscillations (or their harmonics) cannot be detected if the plasma flow velocity is greater than about $\mathrm{L} / \Delta \mathrm{t}(\mathrm{min})=160 \mathrm{~km} / \mathrm{s}$. Since the solar wind velocity is typically about $400 \mathrm{~km} / \mathrm{s}$ it follows that plasma oscillations are not expected to be detected in the solar wind, or in any region where flow velocities exceed approximately $160 \mathrm{~km} / \mathrm{s}$.

[11] The third reason that plasma oscillations might not be detected is that the spacecraft is in a region of plasma temperature so high that the plasma oscillations are extinguished by strong Landau damping. Strong Landau damping occurs when the Debye length is comparable to or greater than the wavelength of the plasma oscillations, i.e., when $\mathrm{k} \lambda_{\mathrm{De}}>1$ [see Gurnett and Bhattacharjee, 2005]. Using $\lambda=40 \mathrm{~m}$ as the characteristic wavelength of the plasma oscillations leads to the following constraint on the electron temperature, $\mathrm{T}_{\mathrm{e}}<8,521 \mathrm{n}_{\mathrm{e}}$, where $\mathrm{T}_{\mathrm{e}}$ is in degrees Kelvin and $\mathrm{n}_{\mathrm{e}}$ is in $\mathrm{cm}^{-3}$. Since the strongest Landau damping is expected to occur at low electron densities (where $\lambda_{\mathrm{De}}$ is large), to estimate the lowest temperature at which Landau damping might be important we use $\mathrm{n}_{\mathrm{e}}=10 \mathrm{~cm}^{-3}$, which is the lowest electron density for which we can expect to detect plasma oscillations. The corresponding temperature is approximately $80,000^{\circ} \mathrm{K}$. This temperature is much greater than the temperatures expected in the Martian ionosphere, so Landau damping is not expected to be an important factor in the ionosphere. However, in the solar wind the electron temperature is typically about $100,000^{\circ} \mathrm{K}$ at the orbit of Mars, so Landau damping is a strong factor in the solar wind, and even more so in the shocked solar wind downstream of the bow shock, where the temperature is even higher. From the above analyses it is apparent that plasma oscillations are not expected to be observed in the solar wind, or in any region where (1) the electron density is less than about $10 \mathrm{~cm}^{-3},(2)$ the flow velocity is greater than about $160 \mathrm{~km} / \mathrm{s}$, or (3) the temperature is greater than about $8,521 \mathrm{n}_{\mathrm{e}}$ degrees $\mathrm{K}$. On the basis of this analysis, we believe that the abrupt disappearance of plasma oscillations (or their harmonics) that is often observed at high altitudes is due to the entry of the spacecraft into the hot, tenuous, rapidly flowing solar wind plasma downstream of the bow shock.

\section{Investigation of Individual Orbits}

[12] Before proceeding with a detailed statistical analysis of the data, it is useful to examine the electron density variations observed during some representative passes. A spectrogram of the electric field spectral density (in $\mathrm{V}^{2} \mathrm{~m}^{2} \mathrm{~Hz}^{-1}$ ) of the received radar echoes as a function of frequency and universal time (UT) is shown in Figure 3 for a 36-min periapsis pass on the dayside of Mars. During this pass the ionospheric sounding data starts at an inbound altitude of $1170 \mathrm{~km}$ and a SZA of $84.57^{\circ}$, extends down to a periapsis altitude of $277 \mathrm{~km}$ at a SZA of $22^{\circ}$, and ends at an outbound altitude of $1142 \mathrm{~km}$ and a SZA of $39.43^{\circ}$. The dark yellow-orange line labeled $\mathrm{f}_{\mathrm{p}}$, in the middle of the spectrogram, is due to the excitation of electron plasma oscillations at the local electron plasma frequency. The second, less bright, yellow-orange line labeled $2 \mathrm{f}_{\mathrm{p}}$ is at the first harmonic of the local electron plasma frequency. The higher harmonics are not well resolved on this spectrogram type of display which averages the intensities over the entire $0-7.31 \mathrm{~ms}$ range of the received time delays. The plasma frequency and the corresponding electron density both reach their largest values in the middle of the spectrogram, near periapsis.

[13] In order to analyze variations in the electron density during a pass such as in Figure 3, we scaled the electron plasma frequency from the harmonics in each ionogram using the electronic ruler (as in Figure 2), and then used equation (1) to compute the electron density. The electron densities are then plotted as a function of time as shown in Figure 4, which is for the same pass as in Figure 3. The vertical axis gives the electron density on a logarithmic 

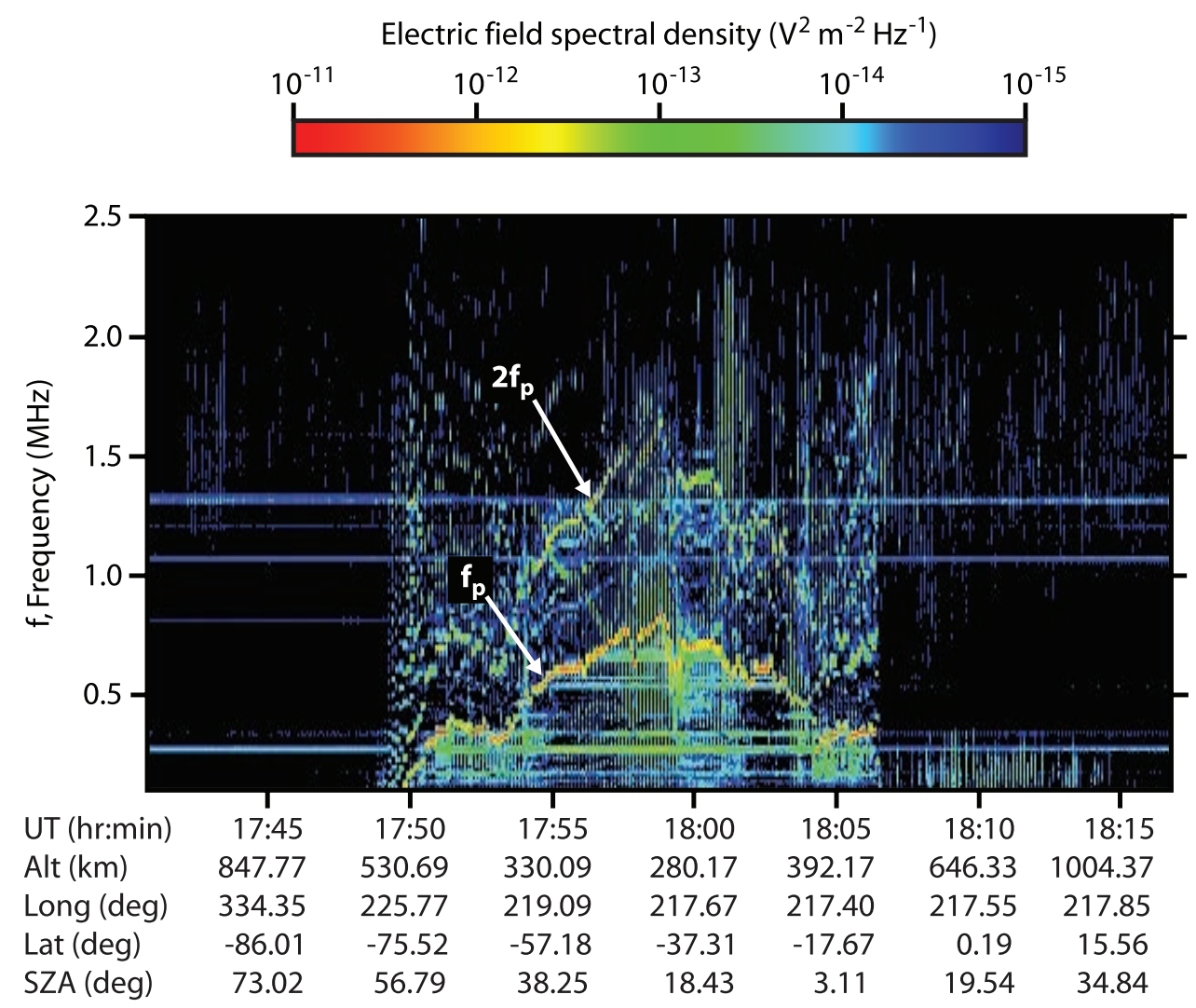

Orbit 2275, October 21, 2005

Figure 3. A display of the radar echo intensity, versus frequency and universal time (UT) (a spectrogram) for a 36 min periapsis pass through the dayside ionosphere. The $x$ axis is also labeled with the altitude, longitude, latitude, and solar zenith angle. The dark yellow-orange line observed at lower frequencies is of the local electron plasma frequency. The second, less bright, yellow-orange line at higher frequencies is the first harmonic of the local electron plasma frequency. The plasma frequency as a function of time is smooth compared to most passes in our data set.

scale and the horizontal axis shows the time in UT and the corresponding altitude, longitude, latitude, and SZA of the spacecraft. Prior to 17:48:46 UT no plasma oscillations (or their harmonics) were detected, suggesting that the spacecraft was in the hot, tenuous shocked solar wind plasma. Starting at 17:48:46 UT plasma oscillations harmonics abruptly appeared with a harmonic frequency spacing corresponding to a density of about $20 \mathrm{~cm}^{-3}$. The plasma oscillation frequencies then rapidly increased and the corresponding electron densities increased to more than $1000 \mathrm{~cm}^{-3}$ in a period of less than one minute. After this initial rapid increase the electron density continued to gradually increase with some relatively small fluctuations as the spacecraft descended in altitude, reaching a maximum of around $8000 \mathrm{~cm}^{-3}$ at periapsis, at about 17:58 UT. As the spacecraft altitude increased after periapsis, the electron density slowly decreased to about $1000 \mathrm{~cm}^{-3}$. At 18:06:30 UT the electron density abruptly decreased to about $20 \mathrm{~cm}^{-3}$ in just one $7.54 \mathrm{~s}$ frequency sweep cycle. After this time no plasma oscillations were observed for the rest of the pass, suggesting that the spacecraft was again in the shocked solar wind plasma. The abrupt changes in the electron density at 17:48:46 UT and 18:06:30 UT and the transitions to ionosphere-like densities between these times are consistent with crossings of the ionopause (i.e., the boundary between the ionospheric and shocked solar wind plasmas [Schunk and Nagy, 2000]). Although the ionopause crossings are clearly defined in this pass, in many cases, such as the next example to be discussed, the ionopause cannot be easily identified.

[14] Although there are some passes where the electron density variations are relatively smooth, as in Figure 4, in most cases the electron density has very large fluctuations on timescales ranging from a few seconds to many minutes. The pass shown in Figure 5 is an example that has such large density fluctuations. In this case electron plasma oscillations were detected over the entire pass, starting with a density of about $30-50 \mathrm{~cm}^{-3}$ at high altitudes, increasing to approximately $9000 \mathrm{~cm}^{-3}$ near periapsis, and then decreasing to about $20 \mathrm{~cm}^{-3}$ at high altitudes near the end of the pass. Although there is a change in the slope at about 18:09 UT, there is no clearly defined density discontinuity that can be clearly identified as an inbound or outbound crossing of the ionopause. The most remarkable feature of this pass, and many others, is the very large density fluctuations that are present during most of the pass. These fluctuations occur on timescales ranging from the smallest timescale that can be measured $(7.54 \mathrm{~s})$ to several minutes, and often have fractional density changes, $\Delta n_{e} / n_{e}$, exceeding a factor of one, sometimes as large as a factor of ten. 


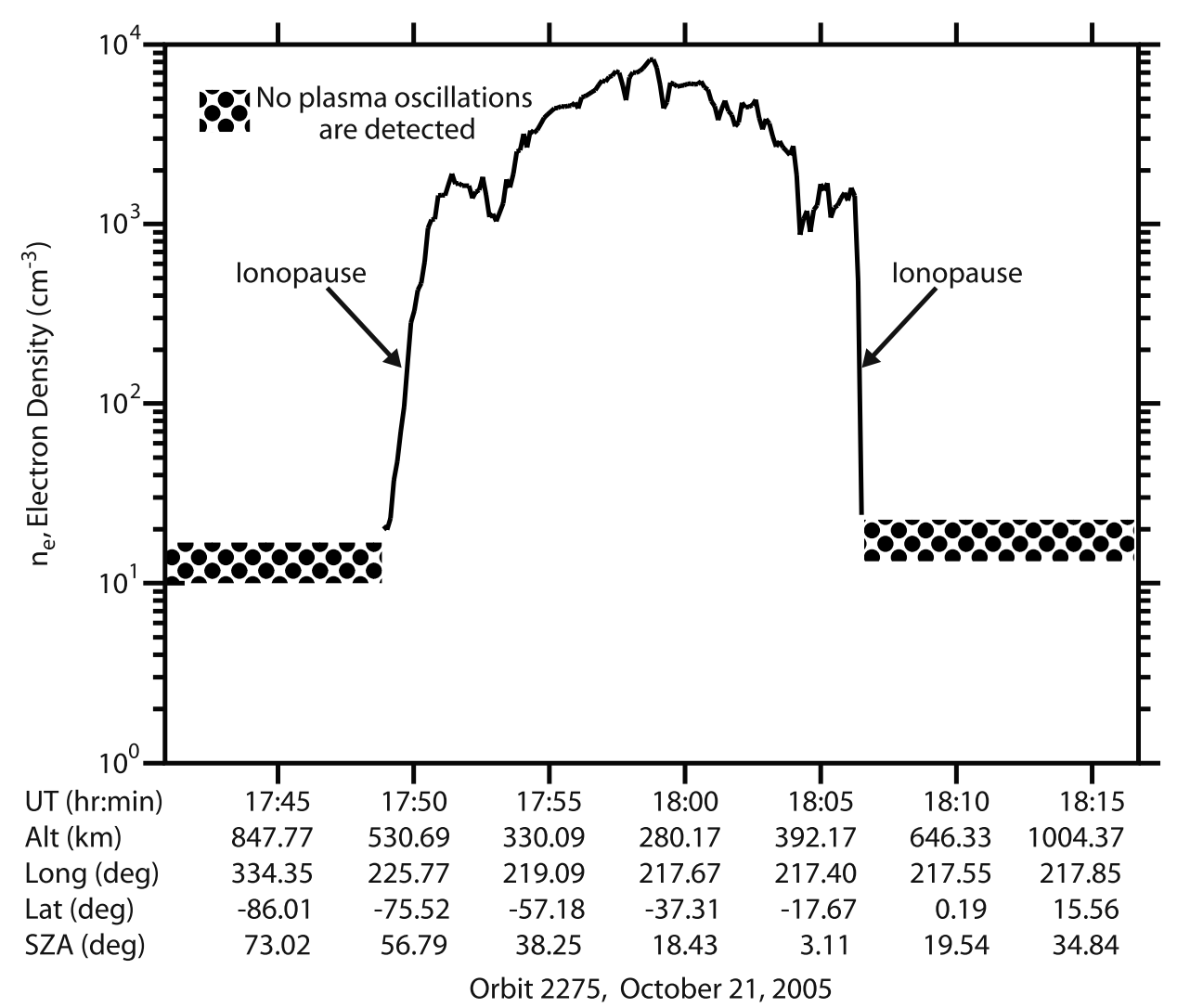

Figure 4. A plot of electron density versus UT for the pass shown in Figure 3. The measurements were made by the method explained in Figure 2. No plasma oscillations are observed from the beginning of the pass at 17:40:51 UT until 17:48:46 UT. The electron density increases as the spacecraft is descending and decreases as it is ascending in a fairly smooth manner. After 18:06:30 UT, the harmonics again are not observed. We infer the position of the ionopause at the start and stop times of the measured plasma frequencies.

Almost $90 \%$ of all the passes studied are of this nature, which means that most of the time the spacecraft is going through regions with highly fluctuating plasma densities. Usually on such passes it is not possible to clearly identify inbound and outbound crossings of the ionopause of the type that are clearly evident in Figure 4.

[15] To investigate whether these large electron density fluctuations have any relationship to the local magnetic field, we have compared these electron density measurements with magnetic field magnitude measurements obtained from electron cyclotron echoes using the technique described by Gurnett et al. [2005, 2008]. No obvious correlation has been identified. In particular, we note that there are regions with highly fluctuating densities for which the magnetic field magnitude variations are smooth, or vice versa. Our preliminary conclusion is that the large highly irregular fluctuations in the electron density are most likely due to an electrostatic shear-driven instability that develops at the boundary between the ionosphere and the rapidly flowing solar wind plasma. The nature and origin of these fluctuations will be discussed in a future paper.

\section{Median Electron Density Versus Altitude}

[16] In order to study the variation in the electron density as a function of altitude and solar zenith angle, plots of the electron density as a function of altitude have been constructed for various SZA ranges. Figure 6 shows a series of such plots for different SZA ranges. These plots show the median electron density, and the lower and upper quartiles (i.e., the 25th and 75th percentile) as a function of altitude. These plots are based only on the ionograms for which the electron density is measurable, i.e., no attempt has been made to correct for the bias introduced by the sparsity of measurable plasma oscillations at high altitudes. Inspection of Figure 6 shows that on the dayside, the logarithm of the electron density decreases with increasing altitude with an almost constant slope, indicating an exponential dependence up to around $750 \mathrm{~km}$. Above this altitude, the slope becomes much steeper. On the nightside, the electron density values are much lower, even at low altitudes, and the change in the slope at around $750 \mathrm{~km}$ is much less obvious. All SZA ranges show substantial fluctuations in the median electron density, with variations sometimes as large as a factor of ten between the $25 \%$ and the $75 \%$ contours. The variability appears to decrease with increasing SZA, particularly in the altitude range around 400 to $600 \mathrm{~km}$. At low altitude, below about $500 \mathrm{~km}$, the fractional fluctuation, $\Delta \mathrm{n}_{\mathrm{e}} / \mathrm{n}_{\mathrm{e}}$, decreases systematically with decreasing altitude, down to only about $\pm 20 \%$ at $300 \mathrm{~km}$.

[17] To determine how large a bias is introduced in Figure 6 by the nondetection of plasma oscillations at high 


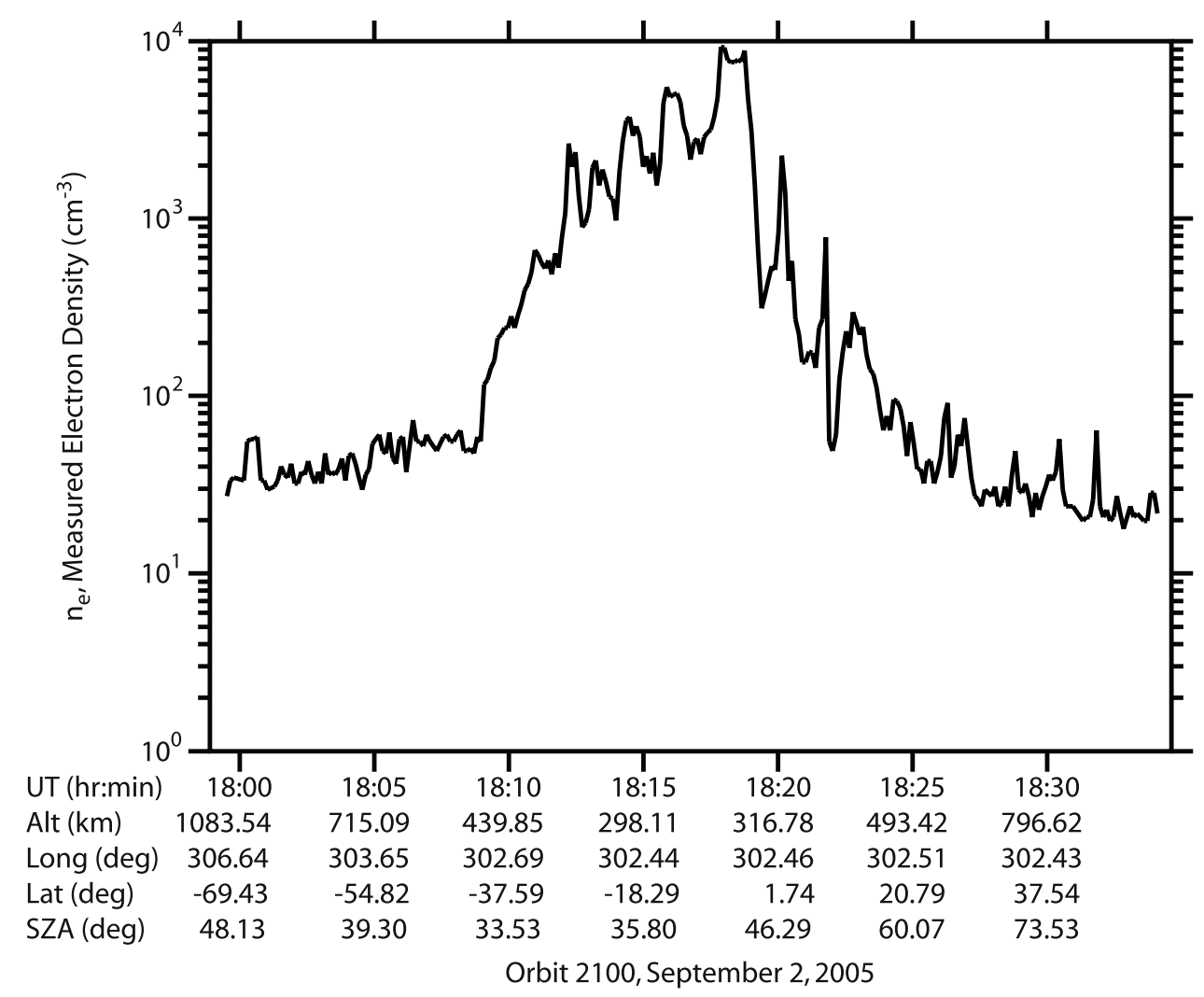

Figure 5. Another plot of electron density versus UT. In this case, as in the majority of passes, the electron density shows a highly fluctuating profile. Small timescale changes in the electron density are observed. Many times, the density changes are more than $100 \%$ between two consecutive samples, $7.54 \mathrm{~s}$ apart.

altitudes, we have investigated the occurrence rate of measurable plasma oscillations as a function of altitude and SZA. The results of this study are given in Figure 7, which shows sampled percentage of the ionograms that show plasma oscillations as a function of altitude and SZA. Five different SZA ranges are shown, corresponding to the ranges shown in Figure 6. It is clear from these plots that the percentage of sampled ionograms with measurable electron densities decreases rapidly with increasing altitude. On the dayside, the fraction of the total data with measurable densities is almost $100 \%$ up to $450 \mathrm{~km}$. This fraction then decreases dramatically with increasing altitude, dropping to less than $10 \%$ in the $0^{\circ}-30^{\circ} \mathrm{SZA}$ range at $1300 \mathrm{~km}$, to $35 \%$ in the $30^{\circ}-60^{\circ} \mathrm{SZA}$ range, and to about $45 \%$, in the $60^{\circ}-90^{\circ}$ SZA range. On the nightside, the percentage with measurable densities at high altitude is much higher, $60 \%$ or more at $1300 \mathrm{~km}$. We believe this trend is due to the fact that the shocked solar wind plasma penetrates to much lower altitudes on the dayside compared to the nightside, where the solar wind plasma is not expected to be encountered at all, even at very high altitudes. The approximate boundary between these two regions is indicated by the dashed line in Figure 7, which goes through the $50 \%$ points for each SZA range.

[18] It is clear from the above analysis that to obtain unbiased electron density profiles as a function of altitude, the ionograms with no measurable plasma frequency must somehow be included in the statistics. To achieve this, we have recomputed the quartiles including all ionograms, even those for which no plasma oscillations (or their harmonics) could be detected. Since a density must be assigned to ionograms for which no plasma oscillations could be detected, we have assumed in these cases that the electron density is less than $10 \mathrm{~cm}^{-3}$. This assumption is justified on the ground that if no plasma oscillations are detected then the spacecraft must be in the hot rapidly flowing shocked solar wind plasma (i.e., with a density less than $10 \mathrm{~cm}^{-3}$, a velocity greater than $160 \mathrm{~km} / \mathrm{s}$, or a temperature greater than $8,521 \mathrm{n}_{\mathrm{e}}$ degrees $\mathrm{K}$ ). As a check on the validity of this assumption we note that since the average electron density of the solar wind near Earth is $8.7 \mathrm{~cm}^{-3}$, and that since the density decreases approximately as $1 / \mathrm{R}^{2}$, where $\mathrm{R}$ is the distance from the Sun [Schunk and Nagy, 2000], then the average solar wind density near Mars should be only about $3.8 \mathrm{~cm}^{-3}$. For such a low solar wind density the plasma oscillation frequency would be too low to detect, even for the shocked solar wind plasma downstream of the bow shock. For unusually high solar wind densities, when the electron density is greater than $10 \mathrm{~cm}^{-3}$, the high velocity and high temperature of the shocked solar wind plasma should still strongly inhibit the occurrence of electron plasma oscillations, still supporting our assumption.

[19] The results of our revised statistical analysis are shown in Figure 8, which is similar to Figure 6, except that the horizontal axis, now labeled "electron density corrected for sampling bias", includes ionograms for which no plasma oscillations could be detected. Unmeasured plasma densities are assumed to be less than $10 \mathrm{~cm}^{-3}$. As can be 


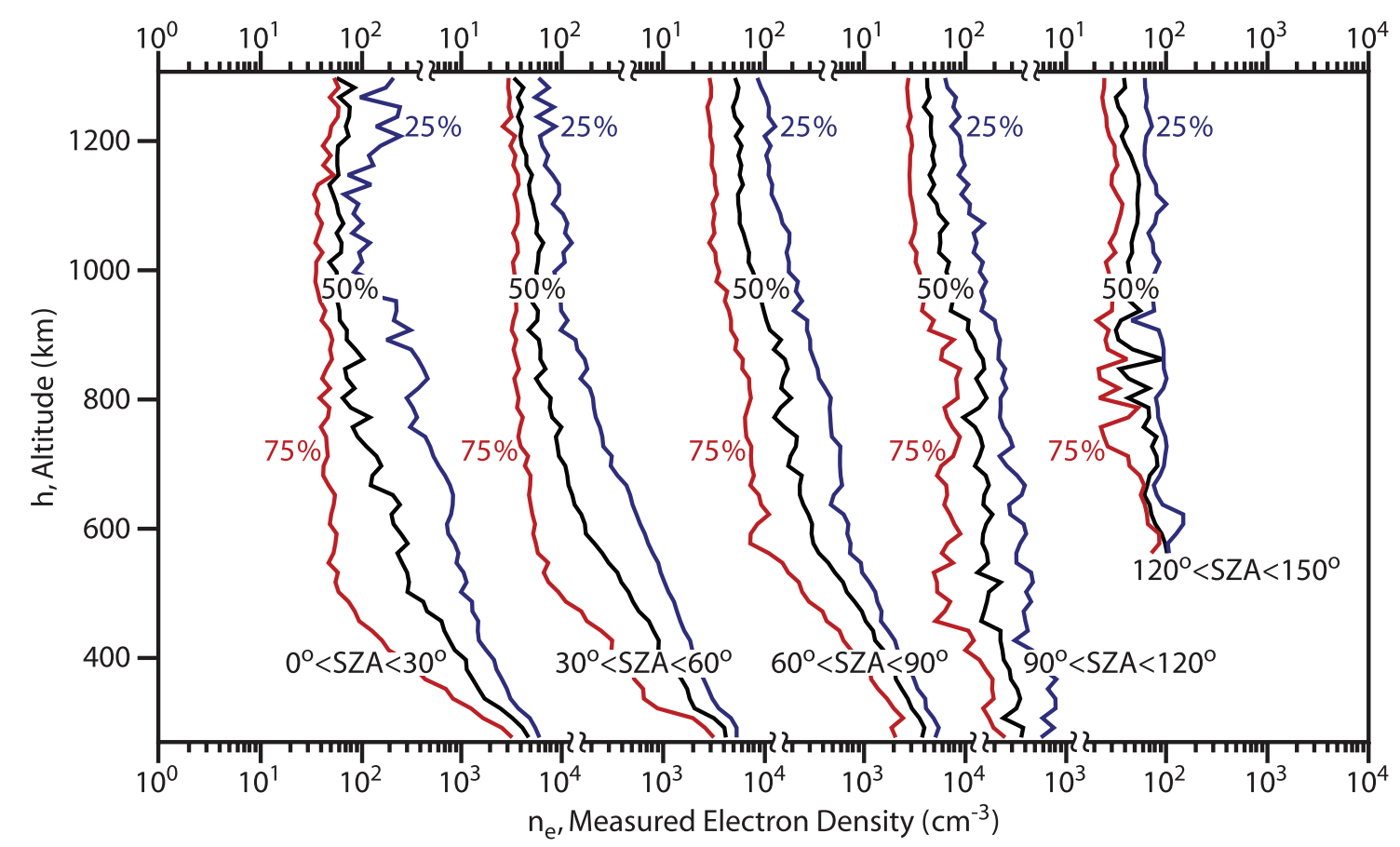

Figure 6. A panel of the measurable electron density profile as a function of the altitude for five different SZA ranges. The $y$ axis represents the altitude and the $x$ axis represents the electron density. Separators are put between different SZA ranges. The black, blue, and red lines are the median, the lower quartile (25th percentile) and the upper quartile (75th percentile), respectively. Samples in which there are no measurable plasma oscillations are excluded from the statistics on which these plots are based. This selection creates a sampling bias. On the dayside, there is an exponential relationship between the average electron density and the altitude up to $750 \mathrm{~km}$. On the nightside, the high altitude densities are about the same as these on the dayside, but the low altitude densities are much less than those on the dayside.

seen the statistics are altered considerably, particularly at high altitude. On the dayside, especially for SZA less than $60^{\circ}$, the median and upper/lower quartile lines terminate at much lower altitudes than in Figure 6. This difference can be easily accounted for by comparing with the percentage of ionograms for which electron plasma oscillations could be detected, as given in Figure 7. Since the percentage of the ionograms with measurable densities is very low at high

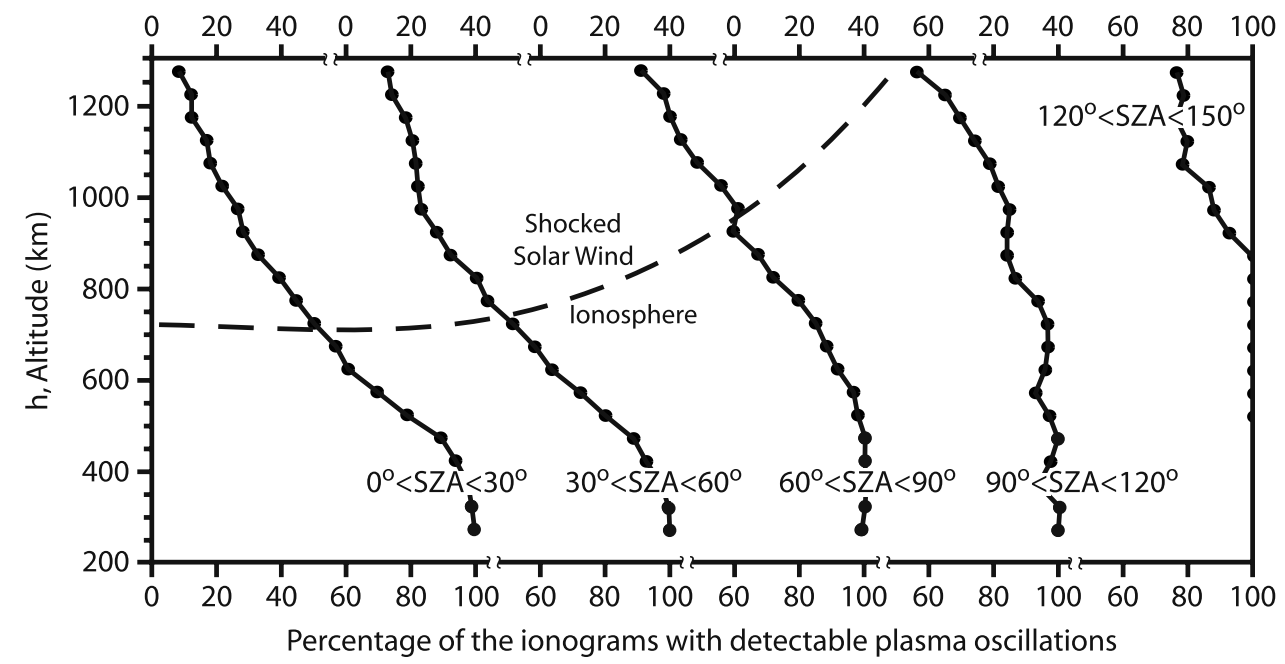

Figure 7. Percentage of the ionograms that display harmonics as a function of altitude, for five different SZA ranges. The measurable sampling percentage increases with increasing SZA and with decreasing altitude. The difference between measurable sampling percentage at high and low altitudes becomes less pronounced as solar zenith angle increases. We believe this can be explained with the fact that the shocked solar wind plasma penetrates to much lower altitudes on the dayside compared to the nightside. The approximate boundary between the shocked solar wind and the ionosphere is indicated by the dashed line, which goes through the $50 \%$ points for each SZA range. 


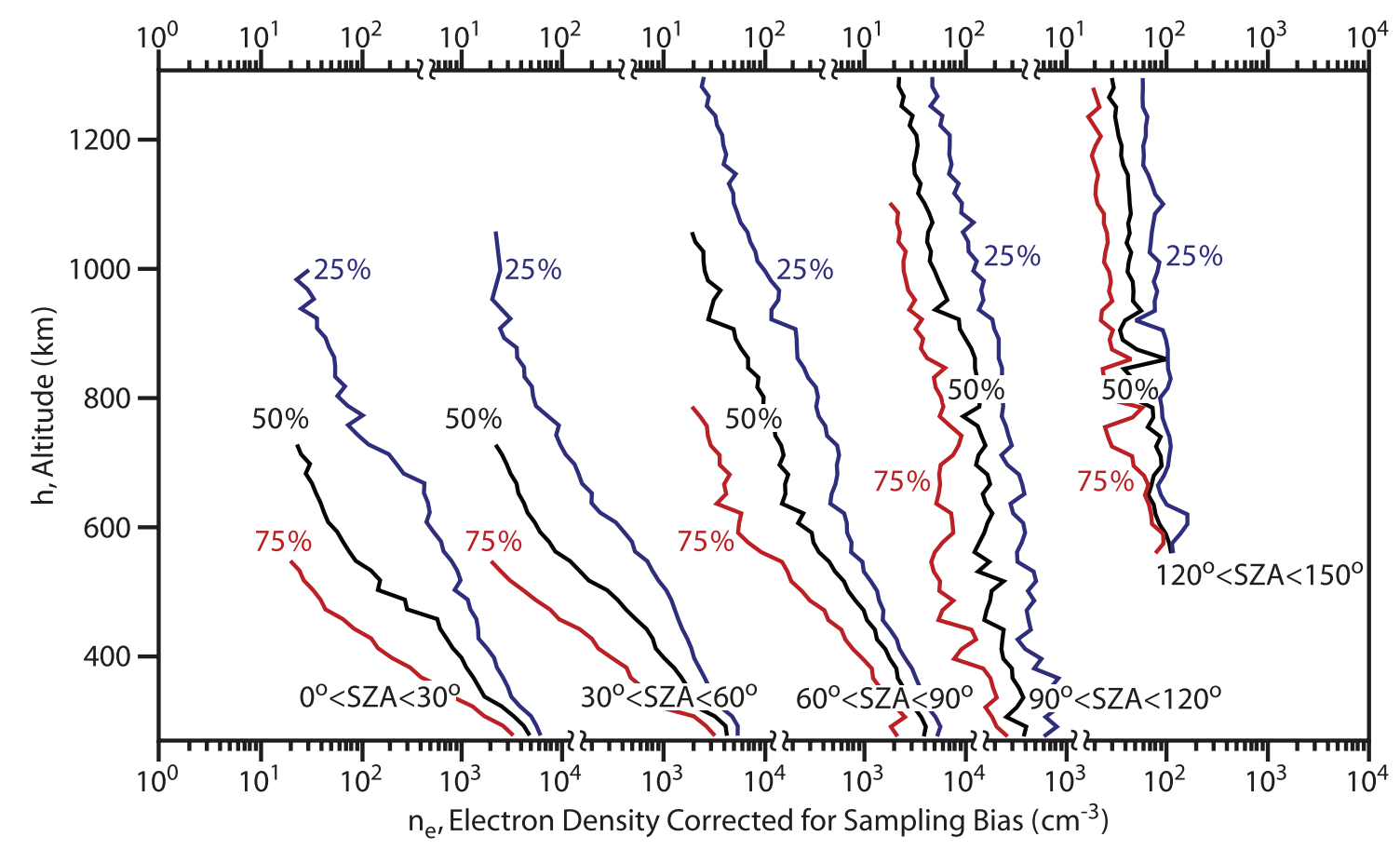

Figure 8. Similar to Figure 6, displaying the electron density profile corrected for sampling bias as a function of altitude for different SZA ranges. Again, the black line, the blue line and the red line show the median, and the lower and upper quartiles, respectively. In this figure, the sample includes cases where the plasma density is unmeasurable. This is done by assuming that when the plasma frequency is not measurable, it is below the lowest measurable electron density, about $10 \mathrm{~cm}^{-3}$. The slope change at around $750 \mathrm{~km}$ is not observed in this plot. The median and the upper and lower quartile lines end at much lower altitudes than those of Figure 6, because these percentiles are below the measurable limit and are therefore meaningless.

altitudes on the dayside, it is impossible to compute meaningful quartiles at these high altitudes. For instance, in the $0^{\circ}-30^{\circ}$ SZA range above $1000 \mathrm{~km}$, only $20 \%$ of the data have density measurements, implying that percentiles above $20 \%$ are not meaningful. However, on the nightside, since the percentage of ionograms with measurable densities remains reasonably high even at high altitudes, the quartiles extend up to the highest altitude $(1300 \mathrm{~km})$ for which data were obtained. Note also, that the change in the slope at about $750 \mathrm{~km}$ evident on the dayside in Figure 6, is now nearly eliminated. Clearly this change in slope was mainly due to the bias imposed by the low occurrence rate of measurable plasma oscillations at high altitudes. It is now apparent that there is an approximate exponential relationship between the total electron density and altitude for all SZA. The e-folding height for this exponential dependence varies considerably, from about 80 to $145 \mathrm{~km}$ on the dayside to more than $1000 \mathrm{~km}$ on the nightside. If this e-folding height is assumed to be the plasma scale height, $\mathrm{H}_{\mathrm{p}}=2 \mathrm{kT} /$ $\mathrm{mg}$, the corresponding temperature on the dayside is between 285 and $520 \mathrm{~K}$, assuming that the ions at these high altitudes are mainly $\mathrm{O}^{+}$and that the variations of gravity with altitude are ignored [Schunk and Nagy, 2000].

\section{Median Electron Density Versus Solar Zenith Angle}

[20] To gain a further understanding of the spatial distribution of the electron density at high altitudes, we have investigated the electron density as a function of the solar zenith angle in a series of fixed altitude ranges. For example, Figure 9 shows the median value of the measured electron density as a function of SZA for the altitude range from 300 to $350 \mathrm{~km}$. The error bars indicate the $25 \%$ and $75 \%$ quartiles for each point. Similar plots have been investigated for $50 \mathrm{~km}$ intervals from $250 \mathrm{~km}$ to $1300 \mathrm{~km}$. Figure 9 shows that on the dayside the median electron density at 300 to $350 \mathrm{~km}$ remains almost constant at about of $2500 \mathrm{~cm}^{-3}$ for SZAs up to about $80^{\circ}$. Beyond about $80^{\circ}$, near the terminator, the density decreases very rapidly going into the nightside. For example, at SZA $=110^{\circ}$ the density in this altitude range has decreased from $2500 \mathrm{~cm}^{-3}$ to about $200 \mathrm{~cm}^{-3}$. The nearly constant electron density on the dayside and the abrupt decrease starting around the terminator occur at all altitudes up to $550 \mathrm{~km}$. Figure 10 shows results for altitudes from 900 to $950 \mathrm{~km}$, where the electron density is much lower, on the order of a few hundred $\mathrm{cm}^{-3}$, and the decrease in density near the terminator is not as evident. At high altitudes, the difference between dayside and nightside density vanishes. Inspection of Figure 8 also shows that at low altitudes, around $300 \mathrm{~km}$, the fractional fluctuations tend to be much smaller than at high altitudes.

[21] The vertical error bars in Figures 9 and 10, which indicate the $25 \%$ and $75 \%$ quartiles for measured values of the electron density, show that the electron densities fluctuate a great deal, not only within a given pass, but also from pass to pass. There can sometimes be a two order of magnitude difference between the minimum and maximum 


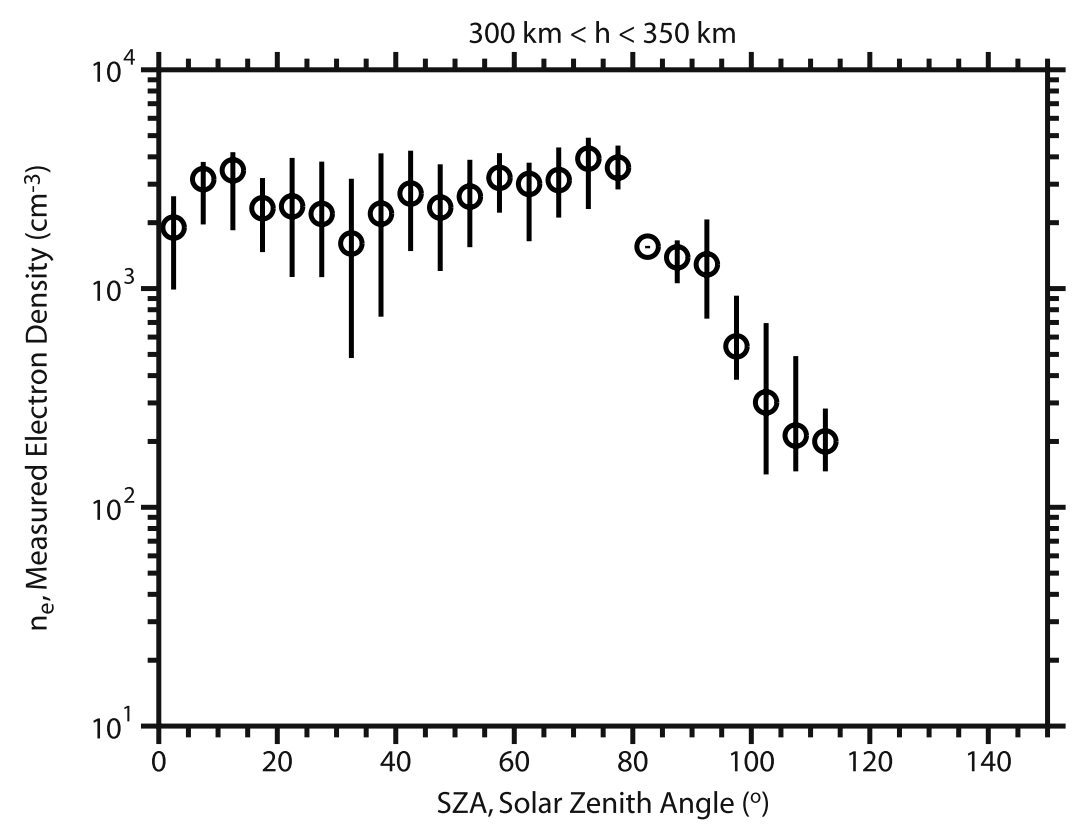

Figure 9. Electron density profile as a function of the solar zenith angle at an altitude range between 300 and $350 \mathrm{~km}$. The median electron density is almost constant on the dayside up to $80^{\circ}$, at around $2500 \mathrm{~cm}^{-3}$. As the spacecraft enters the nightside, there is a substantial decrease in the average electron density. The vertical lines around the data points, which show the lower and upper quartiles at a given SZA, show that the electron density values can vary on the order of $100 \%$.

densities at a given altitude. The nature of these extreme variations can be illustrated by using percentage occurrence plots, such as in Figure 11. This plot gives a color-coded display of the occurrence rate of events within a given electron density range, normalized for each bin in SZA, as a function electron density and SZA. For the altitude range shown $(250-300 \mathrm{~km})$, the occurrence is highest, around
$35 \%$, at densities of approximately $5000 \mathrm{~cm}^{-3}$ throughout the dayside. The highest occurrence rate drops to much smaller electron densities on the nightside. At SZA $=100^{\circ}$, the highest occurrence $(\sim 35 \%)$ is at around $250 \mathrm{~cm}^{-3}$. A comparison of these occurrence rate plots with the average electron density versus SZA plots (as shown in Figures 9 and 10) shows that the electron densities with the maximum

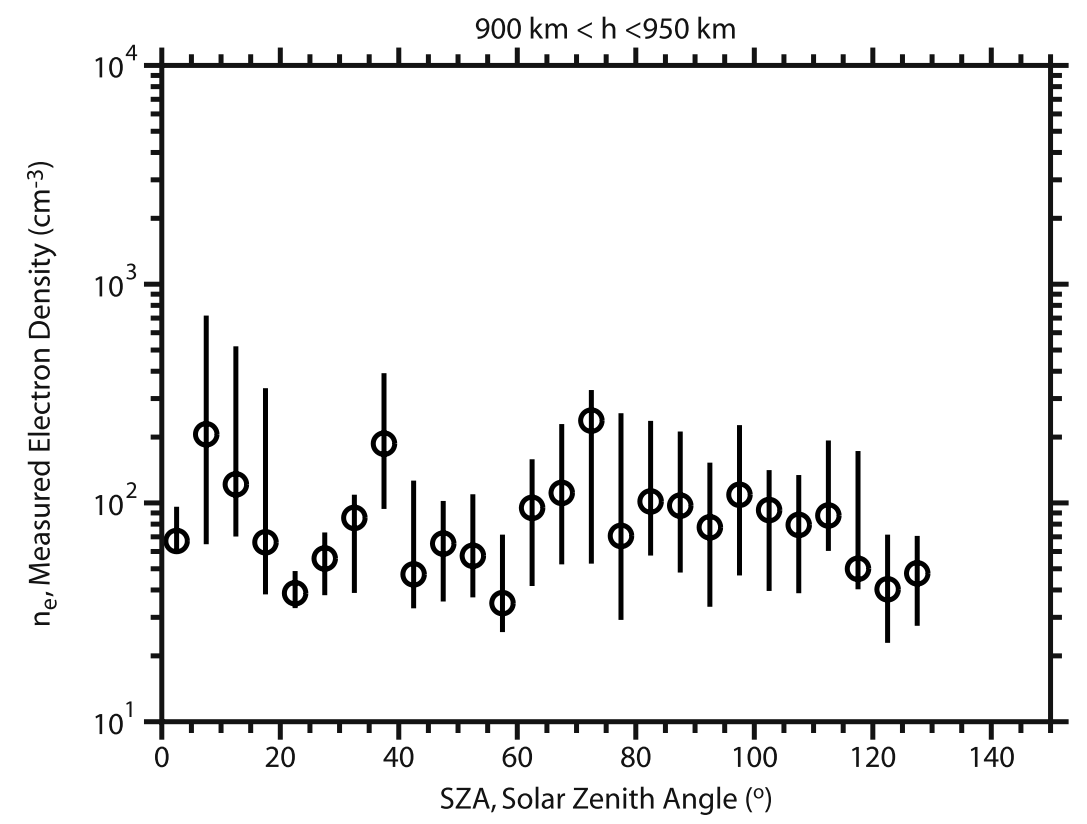

Figure 10. Electron density profile as a function of the solar zenith angle at the altitude range between 900 and $950 \mathrm{~km}$. At high altitudes, the electron density becomes very weak even in the dayside. The dayside densities are quite variable and the difference between the dayside and the nightside vanishes. 


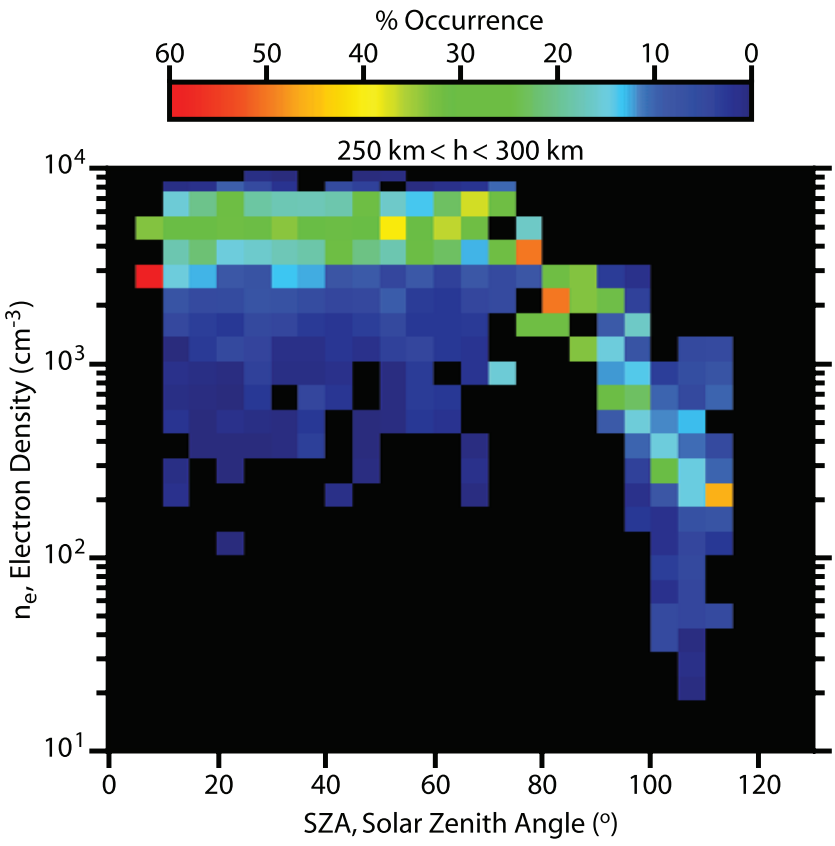

Figure 11. A color-coded display showing the occurrences of the events with given electron density values for the altitude range between 250 and $300 \mathrm{~km}$. The $y$ axis shows the electron density and the $x$ axis shows the solar zenith angle. The percentage occurrence, normalized with each solar zenith angle bin, is given by the color bar. Throughout most of the dayside (between $10^{\circ}$ and $80^{\circ}$ ), the highest occurrence is around $35 \%$ at a density of approximately $5000 \mathrm{~cm}^{-3}$. This value is very close to the average electron density in the dayside. At very low SZA values, the highest occurrence is above $50 \%$, at around 3000 $\mathrm{cm}^{-3}$. The highest percentage occurrence electron density drops to smaller values in the nightside.

occurrence and the median electron density values are in good agreement with each other. In most cases, the maximum occurrence bin is within the $25 \%$ and $75 \%$ lines given in Figure 8. On the dayside, a few events occur with strikingly low electron densities at these low altitudes, sometimes as low as $100 \mathrm{~cm}^{-3}$, even though the median electron density is $5000 \mathrm{~cm}^{-3}$.

[22] To investigate the possible relationship between these density variations and the crustal magnetic field of Mars, the variations in the southern and northern hemispheres have been compared. The northern hemisphere, which has very few regions of strong crustal magnetic field, and the southern hemisphere, where most of the crustal magnetic field regions are located, have comparable variation in the electron density. Because the sample interval covers periods with variations due to seasonal changes, heliocentric distance of Mars, solar activity, solar wind pressure, etc., it is likely that any minor control due to the crustal magnetic field is averaged out. Also, since most of our measurements are from relatively high altitudes, the crustal magnetic fields in the vicinity of the spacecraft are expected to be relatively small and have little influence at these altitudes. However, other more complicated processes involving crustal magnetic fields could still be involved. For example, reconnection with the crustal fields on the dayside could affect the entire dayside solar wind interaction boundary as the reconnected flux tubes are swept over and through the upper ionosphere into the wake.

\section{Discussion}

[23] This study shows that in the altitude range from about 275 to $1300 \mathrm{~km}$ the median electron density in the Martian ionosphere decreases approximately exponentially with increasing altitude with a plasma scale height that varies from about 80 to $140 \mathrm{~km}$ on the dayside to over $1000 \mathrm{~km}$ on the nightside. In the altitude range around 300 to $350 \mathrm{~km}$ the median electron density on the dayside is nearly constant at about $2500 \mathrm{~cm}^{-3}$ for SZAs up to about $80^{\circ}$. Beyond $80^{\circ}$ the density rapidly decreases to $200 \mathrm{~cm}^{-3}$ or less. Large fluctuations from these median values are almost always observed, particularly at higher altitudes on the dayside, often with variations of a factor of two, and sometimes a factor of ten or more. These density fluctuations do not appear to have any obvious correlation to variations in the local magnetic field magnitude. On the dayside the fractional amplitude of the density fluctuations decreases to only about \pm 20 percent at an altitude of $275 \mathrm{~km}$. Although the expected density discontinuity at the ionopause is sometimes observed, in most cases the ionopause cannot be clearly identified due to the very large density fluctuations that are usually present.

[24] It is apparent that the Martian ionosphere, above $300 \mathrm{~km}$, is highly dynamic and is controlled by quite different physical processes than occur in the lower levels of the ionosphere. To understand some of these processes, we have compared our density measurements with two ionospheric simulation models: (1) a three dimensional (3D) hybrid (kinetic-fluid) model, and (2) a three dimensional magnetohydrodynamic (MHD) model. In the hybrid model, a fluid description is used for the electrons and a fully kinetic description is used for the ions. The particles and fields in the hybrid model are treated self-consistently, and the particles are represented by a set of macroparticles which obey the laws of motion for point particles. For a detailed description of the hybrid model see Modolo et al. [2005]. In contrast with the hybrid model, the MHD model is a four-species, high-radial-resolution simulation, which uses the ideal MHD equations to describe the motion of the electrons and ions. For a detailed description of this model, see $M a$ et al. [2004].

[25] The results from these two models are compared with our density measurements in Figure 12. This figure shows the electron density as a function of SZA for an altitude range from 400 to $450 \mathrm{~km}$. The open black circles are the median of the measured local electron density from the MARSIS data, the green line represents the result of the MHD model simulations at $425 \mathrm{~km}$, and the red and the orange lines are from two different hybrid simulations for the altitude range from 400 to $450 \mathrm{~km}$. The hybrid simulation results shown by the red line (labeled omnidirectional) give the density profile averaged over all possible directions of the interplanetary magnetic field (IMF) such that all possible directions are included with the same probability. This model assumes that the average density is azimuthally symmetric around the Mars-Sun line. The hybrid simulation 


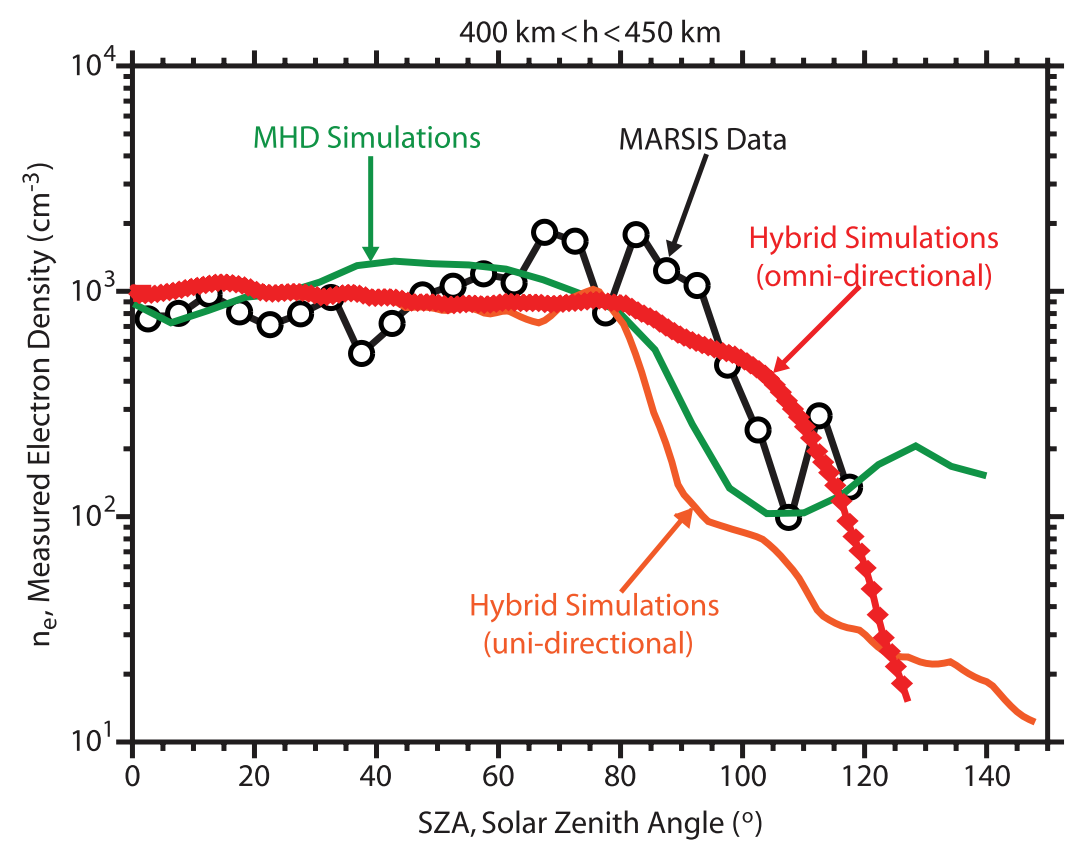

Figure 12. A plot of the electron density versus solar zenith for the altitude range from 400 to $450 \mathrm{~km}$ showing the MARSIS data along with results from hybrid and MHD simulations. The black circles represent the median electron density values from MARSIS data. The green line shows the densities from the MHD simulations, and the red and orange lines show densities from two hybrid simulations. For the red line (labeled omni-directional), all possible directions for the interplanetary magnetic field (IMF) are averaged, whereas, for the orange line (labeled unidirectional), the densities in the plane of the motional electric field $\mathbf{E}_{\mathrm{conv}}=-\mathbf{v}_{\mathrm{SW}} \times \mathbf{B}_{\mathrm{IMF}}$ are given. In all the cases, the electron density is almost constant throughout the dayside and decreases rapidly on the nightside.

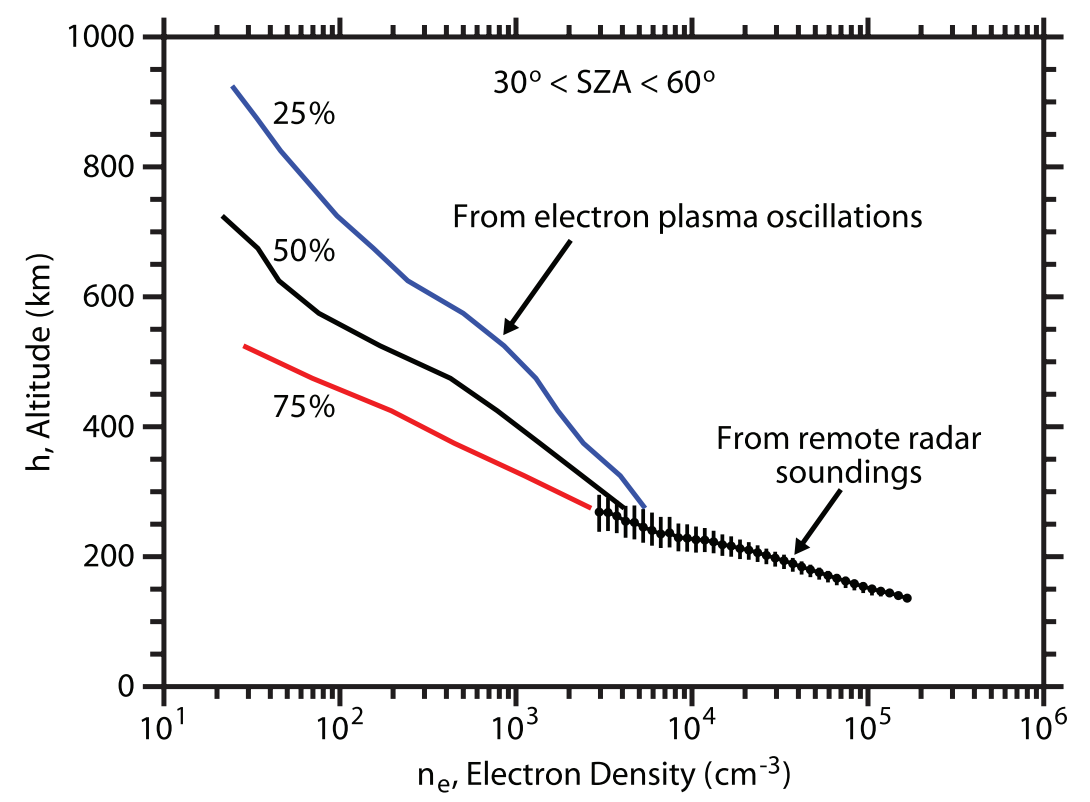

Figure 13. A plot of median electron density with altitude, including the results of ionospheric sounding, corrected for dispersion, and the local measurements described in this paper. The altitude is on the $y$ axis and the electron density is on the logarithmic $x$ axis. The black, red, and blue lines represent the median, 75th percentile, and 25th percentile values of the total local electron density, respectively. The black circles are the electron density values obtained from sounding. Vertical bars show the 75 th and 25 th percentile range in altitude for each density bin for the remote sounding densities. Since sounding covers an altitude range between 130 and $300 \mathrm{~km}$, there is only a small interval where the two data sets overlap. The sounding and local electron density data are in good agreement where they overlap. The slope from local electron density measurements is steeper than that from sounding. We believe that this reflects a transition from a photo-chemical to a transport dominated physical regime. 


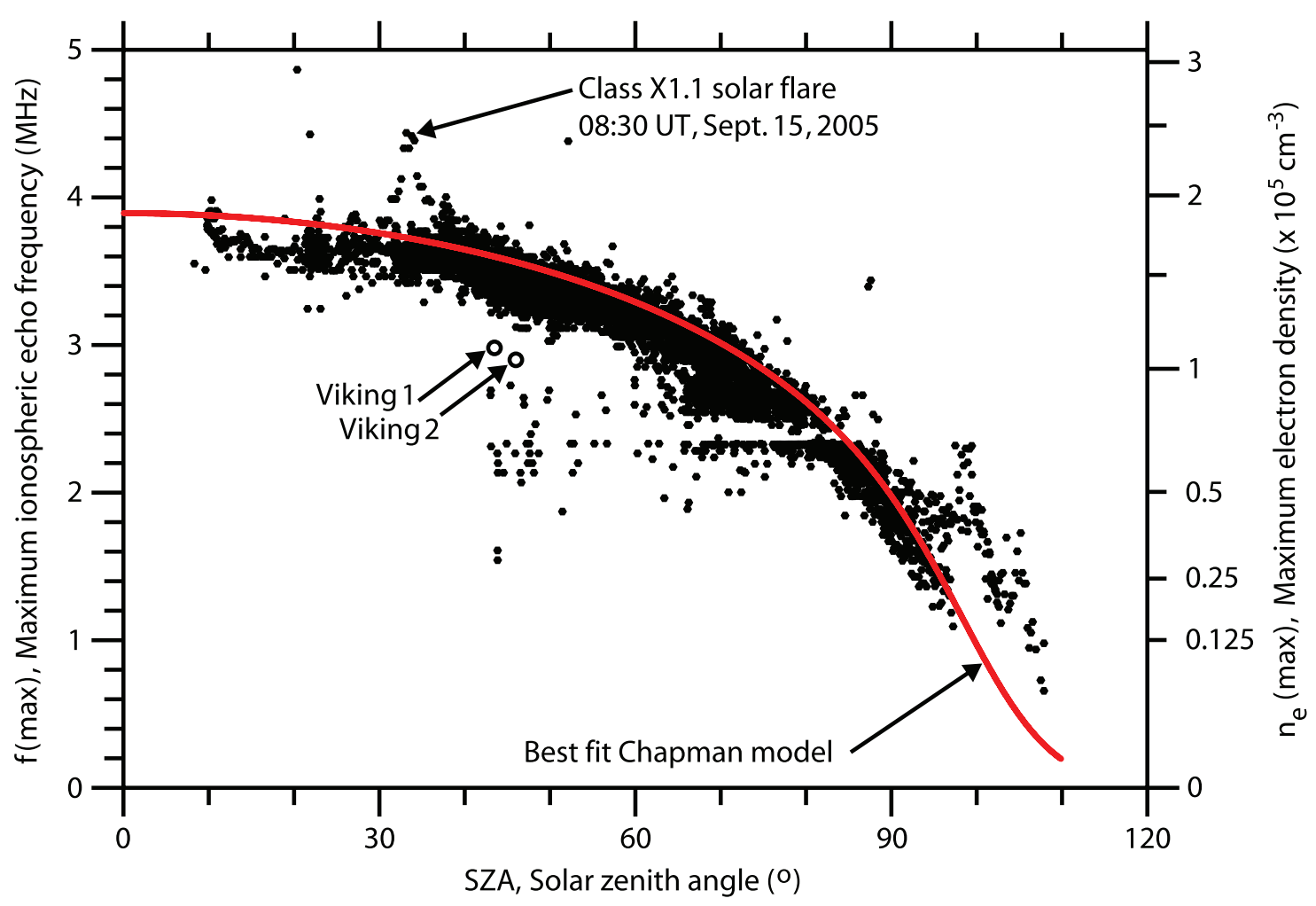

Figure 14. Electron density values obtained by ionospheric sounding are compared to the Chapman model [Chapman, 1931a]. In this plot from [Gurnett et al., 2005], the maximum ionospheric echo frequency is displayed as a function of the SZA. The maximum plasma frequency, and thus the maximum electron density in the ionosphere, decreases with increasing SZA. This result is in sharp contrast to plasma density measurements presented in Figures 9 and 10, where the density is roughly constant over the dayside for a given altitude. We believe this to be evidence of the transition from a photo-chemical to a transport-dominated region.

results shown by the orange line (labeled unidirectional) give the average density profile along the direction of the motional electric field, given by $\mathbf{E}_{\mathrm{conv}}=-\mathbf{v}_{\mathrm{sw}} \times \mathbf{B}_{\mathrm{IMF}}$. Over long time intervals, the average IMF direction is expected to coincide with the direction of the Parker spiral [Parker, 1958]. Since the actual IMF direction is expected to fluctuate around the Parker spiral direction, we expect the observed density profile to lie somewhere between the predictions given by the omnidirectional and unidirectional models (i.e., between the orange and red lines). As can be seen in Figure 12, there is generally good agreement between the median densities from our measurements and the simulations. In particular, the nearly constant electron density at about $1000 \mathrm{~cm}^{-3}$ on the dayside and the rapid decrease going into the nightside are seen to be in reasonable agreement with the observations. However, the unidirectional hybrid and MHD simulations do not agree as well with the observations in the region around the terminator as the omni-directional hybrid simulation.

[26] As Mars Express spacecraft plasma observations [Dubinin et al., 2006] show, and the difference between the omni-directional and unidirectional hybrid simulation models illustrate, a large asymmetry is introduced in the plasma environment due to the influence of the direction of the interplanetary magnetic field. We believe that the large spread in the sampled densities, as indicated by the vertical bars, is largely due to variations in the direction of the interplanetary magnetic field.

[27] It is also interesting to compare our local electron density measurements with electron density measurements obtained from the MARSIS remote radar soundings. Whereas the density measurements from the local plasma oscillations are confined to the altitude range from 275 to $1300 \mathrm{~km}$, the remote sounding measurements [Gurnett et al., 2005, 2008; Morgan et al., 2008] are generally for much lower altitude, between about 130 and $300 \mathrm{~km}$. However, the two data sets overlap around $300 \mathrm{~km}$, so that a reasonable comparison can be made. Figure 13 shows a comparison of the results from the two techniques in the SZA range between $30^{\circ}$ and $60^{\circ}$. The vertical axis shows the altitude and the horizontal axis shows the electron density. The red, black and blue lines give the upper quartile, median, and lower quartile for the local electron density. The solid black dots give the median electron density values obtained from the remote radar soundings. The error bars give the altitude range between quartiles for each density bin. As can be seen the results from the two techniques are in very good agreement in the region around $300 \mathrm{~km}$. However, a definite change in slope can be seen near the transition between the two techniques. This change in slope between 200 and $300 \mathrm{~km}$, is believed to be due to a change in the physical processes that control the vertical 


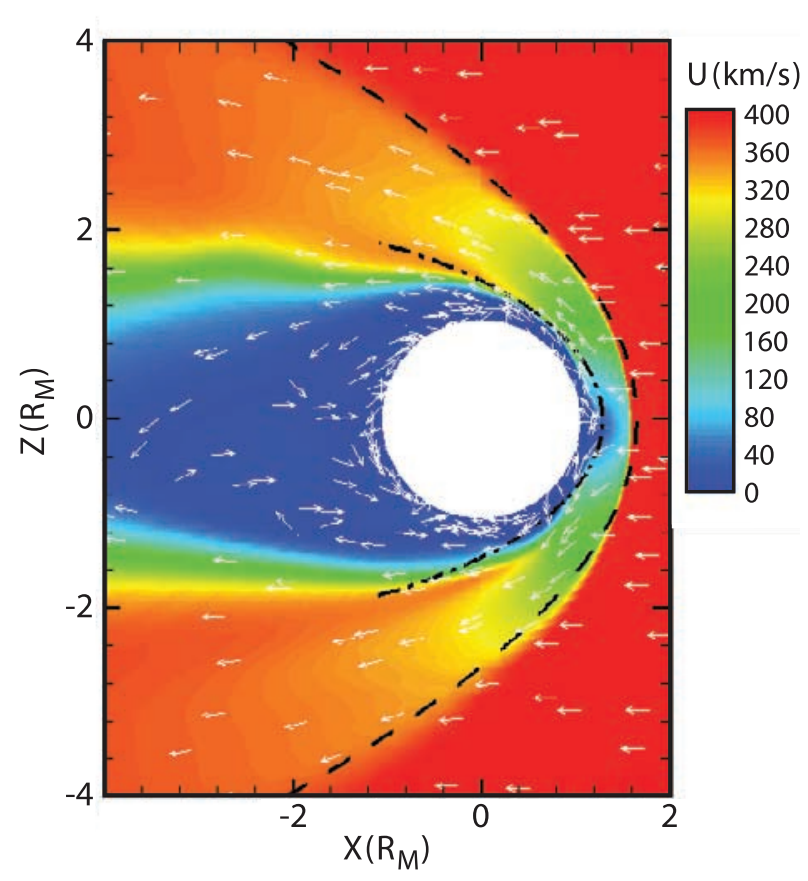

Figure 15. A plot from Ma et al. [2004] showing the velocities calculated with MHD simulations. The white arrows show the direction of the velocities, whereas, the magnitudes of the velocity vectors are shown by the color bar. The subsolar to antisolar flow can easily be seen in this plot, illustrating a possible transport mechanism.

density profile in the two regions. Below $200 \mathrm{~km}$ photochemistry is the dominant process, whereas above $300 \mathrm{~km}$ upward diffusion and horizontal plasma transport become the dominant process. Below $200 \mathrm{~km}$ the Chapman photochemical equilibrium model provides a good first-order approximation to the density profile [Morgan et al., 2008]. As the altitude increases, deviations from the Chapman model start to occur and more complicated models, such as the hybrid and MHD simulation models discussed earlier, must be used to take into account plasma transport. Above about $600 \mathrm{~km}$ there is an obvious further increase in the slope of the measured electron densities (Figure 6) that is probably caused by the occasional entry into the shocked solar wind. As discussed earlier, the reduction in the occurrence rate of plasma oscillations when the spacecraft is in the shocked solar wind causes a bias toward larger densities in this region. We note that this is the same region where magnetic pile-up effects caused by the solar wind become important [Trotignon et al., 2006]. According to Trotignon et al. [2006] the magnetic pile-up boundary is around $850 \mathrm{~km}$ at the subsolar point, increasing to around $1500 \mathrm{~km}$ at the terminator and for SZA from $30^{\circ}-60^{\circ}$, the magnetic pile-up boundary is expected to be between 800 and $1000 \mathrm{~km}$.

[28] It is also of interest to compare the solar zenith angle dependences obtained from the two techniques. Figure 14, from Gurnett et al. [2005], shows a comparison of the electron densities obtained by ionospheric sounding with the Chapman photo-chemical equilibrium model [Chapman, 1931a]. The black dots show the maximum electron densities from radar sounding as a function of SZA in the lower levels of the ionosphere (from about 130 to $150 \mathrm{~km}$ ), and the red line shows the corresponding best fit to the Chapman model. A very clear trend toward decreasing electron density with increasing SZA is apparent. This variation is due to the change in the incidence of the ionizing solar radiation on the atmosphere. The electron density varies approximate as $\cos ^{1 / 2}(\mathrm{SZA})$, exactly as predicted by Chapman's theory. By comparing with Figure 9, which shows the electron density as a function of SZA at 300 to $350 \mathrm{~km}$, one can see that the SZA dependence in the photochemical region at low altitudes is quite different from the SZA dependence observed at the higher altitudes investigated in this study. At high altitude the electron density is almost independent of SZA up to about $80^{\circ}$, and shows no tendency to follow the $\cos ^{1 / 2}$ (SZA) law characteristic of the photo-chemical equilibrium region. The reason for this marked difference is that the Chapman model assumes a completely static ionosphere [Bougher et al., 2001], whereas at the higher altitudes where our measurements were obtained the ionosphere is no longer in static equilibrium. At these higher altitudes the chemical reaction timescales become longer than the transport timescales, so transport becomes the dominant process. Two possible transport effects occur: (1) diffusion, and (2) convection. Above about $200 \mathrm{~km}$, the mean free path becomes greater than the atmospheric scale height. Particles can then diffuse upward away from the region where they are produced. At even higher altitude, the interaction with the shocked solar wind flow causes horizontal convection via the shear stresses imposed on the ionospheric plasma. Plasma simulations show that the resulting horizontal transport explains the nearly constant electron density as a function of SZA on the dayside at an altitude around $300 \mathrm{~km}$ and above. At these altitudes there is a strong antisolar flow away from the subsolar point that is imposed by the interaction of the solar wind streaming around the planet. This flow carries plasma away from the subsolar region toward the terminator, and causes an increase in average electron density at high SZA values and high altitudes. The geometry of this flow can be seen in Figure 15, from Ma et al. [2004], which shows the flow velocities from an MHD simulation. The white arrows give the direction of the flow and the magnitude of the velocity is given by the color. The dash-dot line and the dashed line represent the mean MPB location and the mean bow shock, respectively.

\section{Conclusion}

[29] In this study electron densities from 503 full and nearly full orbit passes have been analyzed, and median electron density profiles were computed over an altitude range from 275 to $1300 \mathrm{~km}$, and a SZA range from $0^{\circ}$ to $140^{\circ}$. The results show that the electron density decreases approximately exponentially with increasing altitude, and that the characteristic plasma scale height that varies from about 80 to $145 \mathrm{~km}$ on the dayside to more than $1000 \mathrm{~km}$ on the nightside. The electron densities at these altitudes are found to be almost independent of solar zenith angle on the dayside, whereas in the photo-chemical equilibrium region 
at the lower altitudes the densities decrease systematically with increasing solar zenith angle, varying approximately as $\cos ^{1 / 2}$ (SZA). Both hybrid and MHD simulations show that the nearly constant electron density as a function of solar zenith angle is caused by horizontal transport of plasma from the subsolar point toward the terminator through interaction with the solar wind.

[30] Our observations also show that the plasma densities are highly variable at all altitudes and solar zenith angles studied. There are relatively few passes with smooth profiles. Although abrupt changes in the plasma density have been observed that can be identified as ionopause crossings, in most cases the ionopause cannot be clearly identified in these data due to the very high level of density fluctuations Although the exact reason for these large fluctuations is not known, we believe that they could be caused by variations in the interplanetary magnetic field direction, which cause asymmetrical variations in the plasma distribution around Mars, by a shear driven instability due to the interaction with the solar wind, by patchy reconnection, or by some combination of these.

[31] Acknowledgments. We would like to thank Pelin Cetin Abaci for her work in processing the ionograms. Research at the University of Iowa was funded by contract 1224107 with the Jet Propulsion Laboratory.

[32] Wolfgang Baumjohann thanks Janet Luhmann and Rudolf Treumann for their assistance in evaluating this paper.

\section{References}

Bougher, S. W., S. Engel, D. P. Hinson, and J. M. Forbes (2001), Mars Global Surveyor Radio Science electron density profiles: Neutral atmosphere implications, Geophys. Res. Lett., 28(16), 3091-3094.

Budden, K. G. (1961), Radio Waves in the Ionosphere, pp. 160-163, Cambridge Univ. Press, New York.

Chapman, S. (1931), The absorption and dissociatival or ionising effect of monochromatic radiation in atmosphere on a rotating Earth, Proc. Phys. Soc., 43, 483

Chicarro, A., P. Martin, and R. Traunter (2004), Mars Express: A European mission to the red planet, Mars Express, The Scientific Payload, SP-1240, edited by A. Wilson, pp. 3-16, ESA Publication Division, Noordwijk, Netherlands.

Dubinin, E., M. Franz, J. Woch, E. Roussos, S. Barabash, R. Lundin, J. D. Winningham, R. A. Frahm, and M. Acuna (2006), Plasma morphology at Mars. ASPERA-3 observations, Space Sci. Rev., 126, 209-238.

Duru, F., D. A. Gurnett, T. F. Averkamp, D. L. Kirchner, R. L. Huff, A. M Persoon, J. J. Plaut, and G. Picardi (2006), Magnetically controlled structures in the ionosphere of Mars, J. Geophys. Res., 111, A12204, doi:10.1029/2006JA011975.

Fjeldbo, G., W. C. Fjeldbo, and V. R. Eshleman (1966), Models for the atmosphere of Mars based on the Mariner 4 occultation experiment, J. Geophys. Res., 71(9), 2307-2316.
Gurnett, D. A., and A. Bhattacharjee (2005), Introduction to Plasma Physics with Space and Laboratory Applications, Cambridge Univ. Press, New York.

Gurnett, D. A., et al. (2005), Radar soundings of the ionosphere of Mars, Science, 310, 1929-1933.

Gurnett, D. A., et al. (2008), An overview of radar soundings of the Martian ionosphere from the Mars Express spacecraft, J. Adv. Space Res., 41(9), doi:10.1016/j.asr.2007.01.062.

Hanson, W. B., S. Sanatani, and D. R. Zuccaro (1977), The Martian ionosphere as observed by the Viking retarding potential analyzers, J. Geophys. Res., 82(28), 4351-4363.

Hinson, D. P., G. L. Tyler, J. L. Hollingsworth, and R. J. Wilson (2001), Radio occultation measurements of forced atmospheric waves on Mars, J. Geophys. Res., 106(E1), 1463-1480.

Kliore, A. J. (1992), Radio occultation observations of the ionospheres of Mars and Venus, in: Venus and Mars: Atmospheres, Ionospheres, and Solar Wind Interactions, Geophys. Monog vol. 66, edited by J. G. Luhmann, M. Tatrallyay, and R. O. Pepin, pp. 265-276, AGU, Washington, D. C.

Kliore, A., D. L. Cain, G. S. Levy, V. R. Eshleman, G. Fjeldbo, and F. D. Drake (1965), Occultation experiment: Results of the first direct measurement of Mars's atmosphere and ionosphere, Science, 149, 1243-1248.

Luhmann, J. G., and L. H. Brace (1991), Near-Mars Space, Rev. Geophys., 29, 121.

Ma, Y., A. F. Nagy, I. V. Sokolov, and K. C. Hansen (2004), Threedimensional, multispecies, high spatial resolution MHD studies of the solar wind interaction with Mars, J. Geophys. Res., 109, A07211, doi:10.1029/2003JA010367.

Modolo, R., G. M. Chanteur, E. Dubinin, and A. P. Matthews (2005), Influence of the solar EUV flux on the Martian plasma environment, Ann. Geophys., 23, 433.

Morgan, D. D., D. A. Gurnett, D. L. Kirchner, J. L. Fox, E. Nielsen, and J. J. Plaut (2008), Variation of the Martian ionospheric electron density from Mars express radar soundings, J. Geophys. Res., doi:10.1029/ 2008JA013313, in press.

Nagy, A. F., et al. (2004), The plasma environment of Mars, Space Sci. Rev., 111, 33-114.

Parker, E. N. (1958), Dynamics of the interplanetary gas and magnetic field, Astrophys. J., 128, 664

Patzold, M., S. Tellmann, B. Hausler, D. Hinson, R. Schaa, and G. L. Tyler (2005), A sporadic third layer in the ionosphere of Mars, Science, 310 837.

Picardi, G., et al. (2004), MARSIS: Advanced radar for subsurface and ionosphere sounding, Mars Express: A European Mission to the Red Planet, SP-1240, pp. 51-70, ESA Division, Noordwijk, Netherlands.

Trotignon, J. G., C. Mazelle, C. Bertucci, and M. H. Acuna (2006), Martian shock and magnetic pile-up boundary positions and shapes determined from the Phobos 2 and Mars Global Surveyor data sets, Planet. Space Sci., 54, 357-369.

Schunk, R. W., and A. F. Nagy (2000), Ionospheres: Physics, Plasma Physics, and Chemistry, Cambridge Univ. Press, New York.

Zhang, M. H. G., J. G. Luhmann, and A. J. Kliore (1990), An observational study of the nightside ionospheres of Mars and Venus with radio occultation methods, J. Geophys. Res., 95(A10), 17,095-17,102.

F. Duru, D. A. Gurnett, R. Modolo, and D. D. Morgan, Department of Physics and Astronomy, University of Iowa, Iowa City, IA 52242, USA. (firdevs-duru@uiowa.edu)

A. F. Nagy and D. Najib, Department of Atmospheric, Oceanic and Space Sciences, University of Michigan, Ann Arbor, MI, USA. 